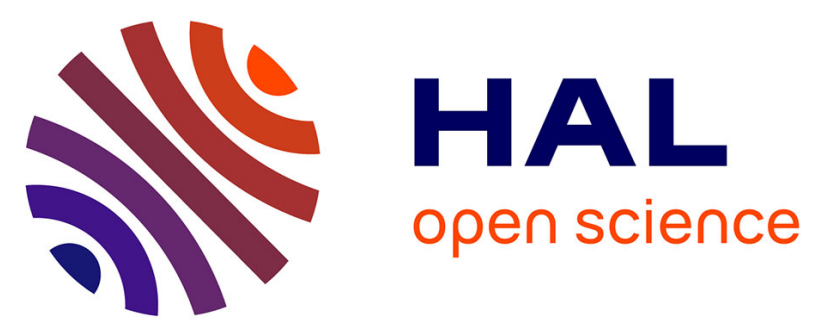

\title{
Synergistic effect for efficient catalytic persulfate activation in conducting polymers-hematite sand composites: Enhancement of chemical stability
}

\author{
Abdellah Ait El Fakir, Zakaria Anfar, Abdallah Amedlous, Asma Amjlef, \\ Salaheddine Farsad, Amane Jada, Noureddine El Alem
}

\section{To cite this version:}

Abdellah Ait El Fakir, Zakaria Anfar, Abdallah Amedlous, Asma Amjlef, Salaheddine Farsad, et al.. Synergistic effect for efficient catalytic persulfate activation in conducting polymers-hematite sand composites: Enhancement of chemical stability. Applied Catalysis A : General, 2021, 623, pp.118246. 10.1016/j.apcata.2021.118246 . hal-03449928

\section{HAL Id: hal-03449928 https://hal.science/hal-03449928}

Submitted on 25 Nov 2021

HAL is a multi-disciplinary open access archive for the deposit and dissemination of scientific research documents, whether they are published or not. The documents may come from teaching and research institutions in France or abroad, or from public or private research centers.
L'archive ouverte pluridisciplinaire HAL, est destinée au dépôt et à la diffusion de documents scientifiques de niveau recherche, publiés ou non, émanant des établissements d'enseignement et de recherche français ou étrangers, des laboratoires publics ou privés. 


\title{
Applied Catalysis A, General, Volume 623, Article Number 118246, AUG 2021 \\ DOI10.1016/j.apcata.2021.118246
}

\author{
Synergistic effect for efficient catalytic persulfate activation in \\ conducting polymers-hematite sand composites: Enhancement of \\ chemical stability
}

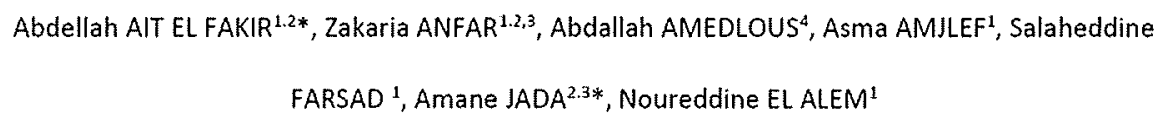

\section{Abstract}

This study explores new chemical strategies for coating the hematite sand (HS) by conductive polymers to enhance the persulfate activation performance. The highest catalytic activity was due to synergistic effect between the HS and the conductive polymers. In fact, conjugated polymers could act as an activator and electronic mediator to promote the conversion of $\mathrm{Fe}$ (III) to $\mathrm{Fe}$ (II). Hence, both conductive polymers used (polyaniline and polypyrrole) have been found to provide the necessary electron transfer, thanks to their nitrogen atoms located in their conjugated chains. The radical scavenging experiments assessed by electronic paramagnetic resonance indicated that the studied pathways nature 
was both a radical and non-radical type. Further, it was found that the protective polymer layers prevented metal ion leaching, mitigate catalyst deactivation, enabled $\mathrm{pH}$ insensitive persulfate activation, and increased the chemical stability. This work provides a new innovative way to remove multiple pollutants in wastewater.

Keyword: Hematite sand (HS), Conductive polymers, Synergistic effect, Persulfate activation, Chemical stability.

\section{Introduction}

Advanced oxidation processes technologies based on the use of sulfate radicals (SRAOPs) have received much attention in the last decades for wastewater treatment [1]. In fact, sulfate radicals $\left(\mathrm{SO}_{4}{ }^{\circ}\right)$, has shown more efficient performance in the degradation of recalcitrant pollutants, due to their high oxidative potentials (2.5-3.1 V) [1-3]. In addition, persulfates (PS) or peroxymonosulfate (PMS), are considered the main sources to generate the $\mathrm{SO}_{4}{ }^{\circ}$ radical $[4,5]$. The activation processes of PS and PMS include many physicochemical approaches that increase the oxidative reactivity of persulfate, as a moderate oxidant $[6,7]$. Therefore, the PS activation involves the formation of highly reactive sulfate radicals $\left(\mathrm{SO}_{4}{ }^{\circ}\right)$ by the $\mathrm{O}-\mathrm{O}$ bond dissociation, and persulfate conversion to $\mathrm{SO}_{4}{ }^{-*}$ by electrons transfer [7]. Among the various activation methods including heterogeneous catalysis (electro or photo), UV photolysis and the use of metal oxide-based catalysts [8]. In addition, iron-based materials have been widely studied as activators of PS, due to the Fe element abundant reserve, their low toxicity, and their very high achievable catalysts in the activation of PS [9-11]. Unfortunately, the inherent drawbacks of iron leaching and the slow conversion of Fe (III) to Fe (II) have resulted in poor catalytic stability of iron oxide, and severely hamper their widespread applications [10]. Furthermore, carbon-based materials or carbocatalysts such as 
graphene oxide, reduced graphene oxide [12-14], nanodiamond carbon nanotubes $[15,16]$, were considered as promising carbocatalysts for the PS activation for organic pollutants degradation. In fact, the unique properties of these metal-free catalysts (e.g., large surface area, superior conductivity, non-toxicity, high catalytic activity, and superior biocompatibility) make them promising candidates in the AOPs $[6,7,17]$. Further, the nitrogen doping in the carbocatalyst structures increases the charge density of adjacent carbon atoms, which promotes the interaction between the catalyst and the PS, facilitating hence the $0-0$ bond cleavage, and also inducing a non-radical degradation pathway of the pollutant [17-19]. It is supposed that the $\mathrm{N}$ atoms can effectively create point defects, which could generate ${ }^{1} \mathrm{O}_{2}$, while oxygen groups in the carbon matrices network are the main active sites contributing to the electrons transfer $[6,7]$. Recently, nitrogen doped carbon-based catalysts-encapsulated metal particles have been shown to promote the activating ability of persulfate in a synergistic and significant manner (Table S1). In fact, transition metals (e.g. iron) coated with nitrogen doped carbon structures could increase the charge density of adjacent carbon atoms. This option favours the interfacial electronic exchange by creating a synergistic effect between the core (transition metals) and the shell (carbon matrix), enhancing hence the electrons exchange between the catalyst and the persulfate and also facilitating the mass recovery of the catalyst after use [20-27]. Moreover, the carbon coated metals catalysts attenuate or probably prevent the metal ions leaching, during the catalytic reaction, and improves hence the chemical stability of metal catalysts $[22,28-30]$. On the other hand, the determination of the dominant pathways during the PS activation, by using these kinds of catalysts, needs to be elucidated (i.e., the incorporation of metal into the carbon matrix may involve the production of either radical or non-radical species, or both). Therefore the origin of the synergistic efficiency occurring between both materials and resulting in the catalytic performance 
improvement and the stability during the recycling tests, must be studied in depth and confirmed $[20,22,28]$.

The present study was carried out in order to fill the knowledge gap regarding the use of metal-carbon composites for persulfate activation. Thus, in our work, nitrogen-rich conductive polymers, such as polyaniline (PANI), and polypyrrole (PPY), were used for the functionalization of rich iron oxide hematite sand (HS) particles. It should be noted that the location of nitrogen atoms in the conjugate chains, improves the polymer stability and prevents its oxidation. Furthermore, PANI and PPy, are electrically conductive polymers, and their conjugated chains can provide the electrons transfer, necessary to create a synergistic effect with the iron metal species. On the other hand, the hematite sand (HS) rich in iron oxide were chosen for their low cost and their abundant in nature. The prepared catalysts HS@PANI and HS@PPy, were used as heterogeneous catalysts to activate the PS for the degradation of organic pollutants to $\mathrm{CO}_{2}$ and $\mathrm{H}_{2} \mathrm{O}$. More, we have used various characterization techniques to verify and to confirm the expected functionalized catalyst structures, and to elucidate the synergistic effect occurring between the hematite sand and the conductive polymers.

\section{Experimental section}

\subsection{Catalysts preparation}

The chemicals and the materials used in this study are listed in Text S1, Supporting Information. The PANI functionalized HS (HS@PANI) catalyst was prepared following in-situ polymerization strategy, and by using common oxidation method of aniline monomer with a known ratio (aniline/ammonium persulfate (APS): 1/1.5). Thus, $1 \mathrm{~g}$ of HS was firstly crushed and screened at $50 \mu \mathrm{m}$ (HS particle size $<50 \mu \mathrm{m}$ ), then dispersed in $50 \mathrm{ml} \mathrm{of} \mathrm{HCl}(0.1 \mathrm{M})$. 
Subsequently, $0.5 \mathrm{ml}$ of aniline was added to the $\mathrm{HS}$ aqueous dispersion, and the resulting mixture was sonicated for $30 \mathrm{~min}$. In the later step, APS ( $1.85 \mathrm{~g}$ dispersed in $50 \mathrm{ml}$ of HCl $(0.1$ M)) was slowly added to the HS-aniline suspension for 2 hours with constant stirring. A dark green color resulted from the HS-aniline-APS mixture which was due to the oxidative polymerization process. At the end of the polymerization reaction, the obtained composite was washed with distilled water and ethanol, then dried at $60^{\circ} \mathrm{C}$ under vacuum for 12 hours. By using the same in-situ polymerization strategy, the polyaniline (PANI) alone was prepared as a control catalyst [31].

Thus, a weighted of HS (HS particle size $<50 \mu \mathrm{m}$ ) amount of $1 \mathrm{~g}$ was dispersed in $50 \mathrm{ml}$ of distilled water. Thereafter, $0.5 \mathrm{ml}$ of pyrrole was added to the HS aqueous dispersion, and the mixture was sonicated for $30 \mathrm{~min}$. In the later step, $\mathrm{FeCl}_{3}, 6 \mathrm{H}_{2} \mathrm{O}(4.38 \mathrm{~g}$ dispersed in $50 \mathrm{ml}$ of distilled water) was slowly added to the HS-pyrrole suspension for 2 hours with constant stirring. As it was expected, the resulting HS-pyrrole-APS mixture took a black color due to the oxidative polymerization process. Finally, the resulted HS@PPy catalyst was washed with distilled water and ethanol, and then dried in an oven at $60^{\circ} \mathrm{C}$ under vacuum for 12 hours. In the same context, the Polypyrrole (PPy) alone was also prepared, as a control catalyst [31].

\subsection{Catalysts characterization methods}

The composition and the surface chemical states of the prepared catalysts were analysed by X-ray photoelectrons spectroscopy (XPS), using XPS SES-2002 (VG SCIENTA). Transmission Electron Microscopy (TEM) type Philips CM 200, 20-200 kV, was used to assess the catalysts nanostructures, whereas their morphologies were analysed by using Scanning Electron Microscopy (SEM) FEI, Model Quanta 400. RAMAN Spectroscopy, type Horiba model Labram BX40, with CCD detector, operating at laser line of $532 \mathrm{~nm}$, was used in this work, to 
determine the molecular composition and the catalysts external structures. The pore size distributions and the specific surface areas were measured using ASAP 2420. To get further insights on the catalysts surface structures, Fourier Transform Infrared (FTIR) spectra were obtained in the mid infrared region $\left(400-4000 \mathrm{~cm}^{-1}\right)$ using a Shimadzu $4800 \mathrm{~S}$. The catalyst structural analyzes were assessed by X-ray diffraction (XRD), and the data were collected on a PANalytical MPD X 'diffractometer, Pert Pro, operating with Cu K $\alpha$ radiation $(\lambda=0.15418 \mathrm{~nm})$, equipped with a real-time multi-band detector X'Celerator (active length $1 / 42.122 \theta$ ).

\subsection{Experimental Procedure and Analytical Methods.}

The catalytic activation of the PS was carried out in a $100 \mathrm{~mL}$ beaker containing $50 \mathrm{~mL}$ of Orange $\mathrm{G}(\mathrm{OG}$ ) solution (50 mg L-1), with constant agitation, by using known concentration of the PS, and the catalyst doses. The initial solution $\mathrm{pH}$ was adjusted with aqueous solutions of $\mathrm{H}_{2} \mathrm{SO}_{4}(0.1 \mathrm{M})$ and $\mathrm{NaOH}(0.1 \mathrm{M})$. The experiments in real conditions were carried out in tap water (TW) under the same conditions. Further, other pollutants were tested, such as Rhodamine B (RhB) and Bisphenol A (BPA), under the same conditions as OG, but with different pollutant concentrations $\left(20 \mathrm{mg} \mathrm{L}^{-1}\right.$ for $\mathrm{RhB}$ and $10 \mathrm{mg} \mathrm{L}^{-1}$ for BPA). After the degradation reaction, the residual organic contaminants concentrations of OG, RhB and BPA, were analysed by using UV-Vis spectroscopy model 6705 UV / Vis JENWAY, at wavelengths of, 478,554 and $278 \mathrm{~nm}$, respectively. To study the mineralization of the different pollutants, the total organic carbon (TOC) was measured, using TOC-L SHIMADZU analyser. In addition, to determine the nature of reactive species produced during the catalytic conversion process, various radical scavengers such as ethanol (EtOH) and tert-butyl-alcohol (TBA), with welldetermined molar ratio EtOH/PS or TBA/PS, were added in the reaction media to trap $\mathrm{SO}_{4}{ }^{\circ}$ and $\mathrm{OH}^{*}$. Further, the sodium azide $\left(\mathrm{NaN}_{3}\right)$ was used as a deactivator for ${ }^{1} \mathrm{O}_{2}$. It should be 
noted that the experimental tests were performed in triplicate, so the error bars were added in figures dealing with the contaminant degradation. Further, in order to identify the presence of the ROS in the degradation process, in situ electron paramagnetic resonance (EPR) analyzes were conducted. Thus, for each analysed solution, a volume of $50 \mu \mathrm{L}$ of the reaction medium was withdrawn and then it was transferred in a glass capillary sealed with Crit-O-seal TM and finally placed in an ESR-tube with an outside diameter of $5 \mathrm{~mm}$. N-tert-butyl- $\alpha$-phenylnitrone (PBN) and 2,2,6,6-teramethypiperidine (TEMP) were used as spin trap agents. Continuouswave EPR spectra were recorded at room temperature and in aerated medium using an EMXplus X-band spectrometer (Bruker). For the stability tests, the catalysts were magnetically separated after each run, thereafter it was washed with ethanol /distilled water three times, and finally dried at $333^{\circ} \mathrm{K}$ for their reuses.

\section{Results and discussions}

\subsection{Catalysts Characterization.}

XPS analyzes, of the prepared HS@PANI and HS@PPy, were performed in order to highlight the catalyst surface chemical functionalities. Thus, after the insertion of HS particles in the polymer's matrix, the wide scan XPS spectra show the presence of the $\mathrm{C}, \mathrm{N}$ and $\mathrm{O}$ chemical elements on the PANI and PPy surfaces, and the Fe element has appeared with a low percentage as resulting from the PANI and PPy layer deposition on the HS particle surface (Fig. S1). Further, the deconvolved C 1s, N 1s and Fe $2 p$ spectra are represented in Figure 1. The deconvolved Fe 2p spectrum for HS@PANI (Fig. 1a) shows two peaks centred mainly at 710.4 and $724.6 \mathrm{eV}$, corresponding to Fe $2 \mathrm{p}_{3 / 2}$ and Fe $2 \mathrm{p}_{1 / 2}$, respectively, and a satellite peak at 719.2 eV corresponds to $\mathrm{Fe}_{2} \mathrm{O}_{3}$ [32-34]. Further, the $\mathrm{C}$ 1s spectrum of HS@PANI (Fig. 1b) can be deconvolved into four principal components at $283.87,285.01,286.29$ and $287.76 \mathrm{eV}$, which 
are mainly due to $\mathrm{C}-\mathrm{C} / \mathrm{C}-\mathrm{H}, \mathrm{C}-\mathrm{N} / \mathrm{C}=\mathrm{N}, \mathrm{C}-\mathrm{O}$ and $\mathrm{C}=\mathrm{O}$ respectively $[33,34]$. The $\mathrm{N} 1 \mathrm{~s}$ spectrum of HS@PANI (Fig. 1C) can be deconvoluted into four peaks at 398.70, 399.96, 401.74 and 403.96 $\mathrm{eV}$, which are mainly due to the $=\mathrm{N}$ - (quinoid imine group), $-\mathrm{NH}$ - (benzoic amine group), $=\mathrm{NH}^{+}-$ (charged amine) and $-\mathrm{NH}_{2}{ }^{+}$- (imine) respectively $[33,34]$. The deconvolution of $\mathrm{Fe} 2 \mathrm{p}$ of HS@PPy is presented in Figure 1d, and it shows the same peaks of HS@PANI (710.4 and 724.6 $\mathrm{eV}$, corresponding to $\mathrm{Fe} 2 \mathrm{p}_{3 / 2}$ and $\mathrm{Fe} 2 \mathrm{p}_{1 / 2}$ and the satellite peak at 719.2 ). In addition, the deconvolution of C 1s for HS@PPy (Fig. 1e) consists mainly of three components at 288.43, 286.80 and $284.87 \mathrm{eV}$, corresponding, respectively, to $\mathrm{C}-\mathrm{O}, \mathrm{C}-\mathrm{N}$ and $\mathrm{C}-\mathrm{C}$ bonds $[35,36]$. The $\mathrm{N}$ 1s spectrum of HS@PPy (Fig. 1f) can be deconvolved into three peaks centred at 398.2, 399.8 and $400.786 \mathrm{eV}$, and corresponding, respectively, to $=\mathrm{N}^{\delta+} / \mathrm{C}-\mathrm{N}^{\delta+}$ (positively charged nitrogen group), pyrrolic $\mathrm{N}(-\mathrm{N}-\mathrm{H})$ and nitrogen bond $(=\mathrm{N}-)[35,36]$.

(a)

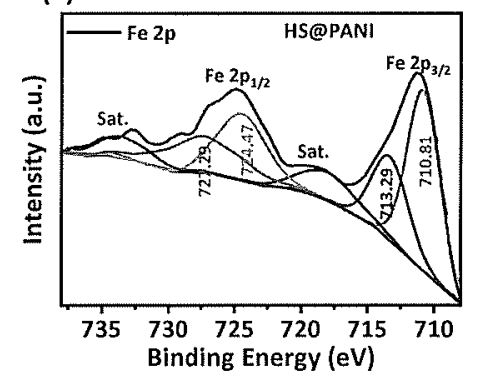

(d)

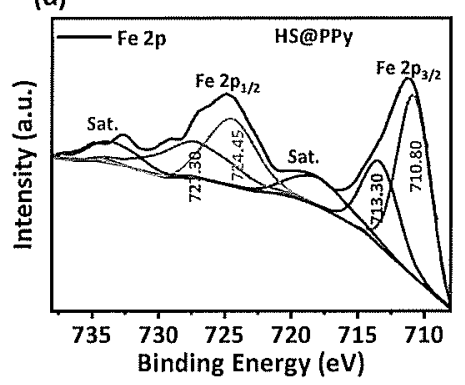

(b)

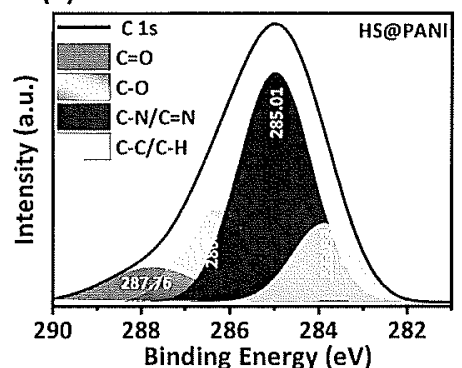

(e)

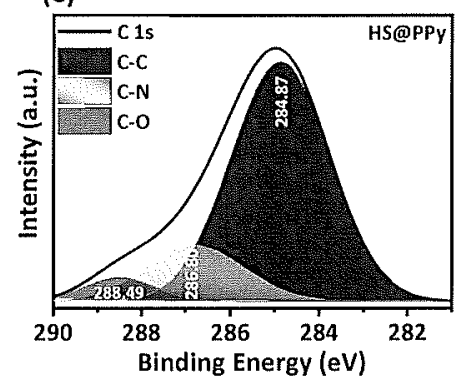

(c)

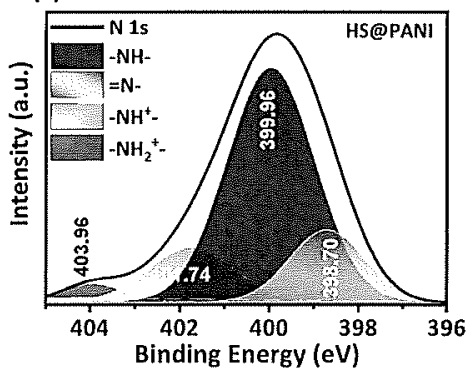

(f)

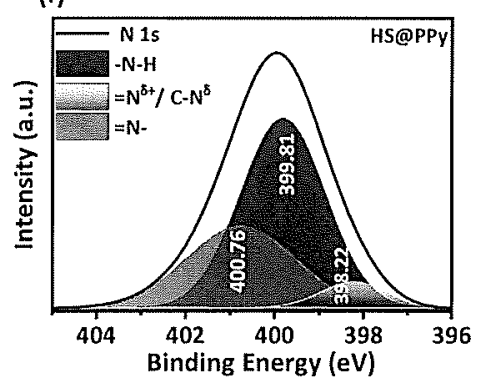

Fig. 1. High-resolution XPS spectra Fe 2p(a), C 1s (b)and N 1s(c) of HS@PANI; Fe 2p(d), C 1s

(e) and N 1s(f) of HS@PPy 
To observe the morphologies and the nanostructure natures of HS, PANI, PPy, HS@PANI and HS@PPy materials, we carried out SEM and TEM analyzes. Thus, as can be observed in Figure S2, the HS particles are irregularly shaped, and their sizes show polydispersity. On the other hand, the TEM images show the PANI and PPy morphologies and reveal the presence of, respectively, irregular, and spherical nanometric particles. However, upon covering of HS particles by PANI and PPy polymers, it can be seen in Figures a-d, that small HS particle sizes are indeed encapsulated inside the polymer matrix. Moreover, PANI and PPy layers attached on the surface of HS particles have been clearly seen. The polymer layers were thinner. In fact, HS particles are visible as darker dots within the brighter polymer chains. The SEM and TEM results confirm the successful HS encapsulation by the polymer and the formation of the molecularly engineered hematite sand/conductive polymer composite.

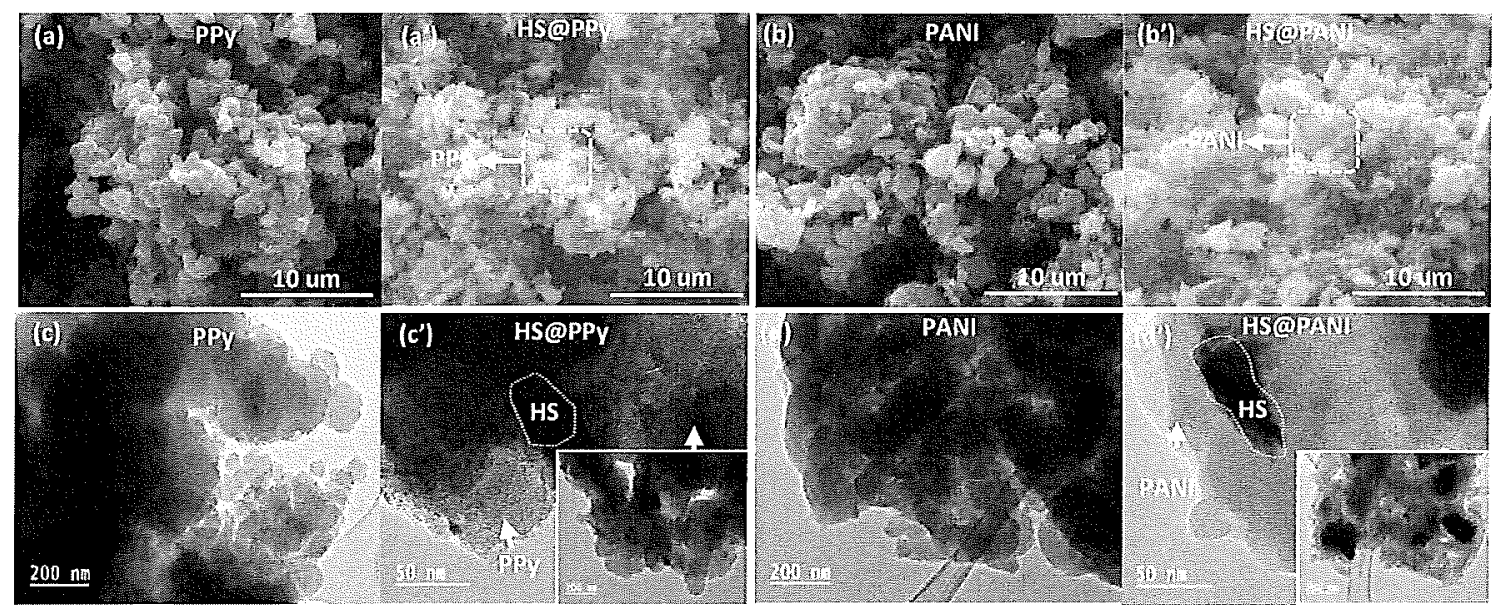

Fig. 2. SEM and TEM images of $(a, c)$ PPy and HS@PPy; $(b, d) P A N I$ and HS@PANI.

To study deeply the structural and the chemical functionalities of the prepared catalysts (HS@PANI and HS@PPY), FTIR analyzes were performed as depicted in Figures 3 a, b. Hence, the spectrum of PPy alone (Fig. 3a) shows that characteristic bands appearing at 1554 and $1466 \mathrm{~cm}^{-1}$ which are attributed to the $\mathrm{C}=\mathrm{C}$ stretch of the PPy ring. The bands located at 1316 
and $1180 \mathrm{~cm}^{-1}$ are due to the $=\mathrm{C}-\mathrm{H}$ bond. In addition, the band centered at $910 \mathrm{~cm}^{-1}$ is due to the asymmetric stretching of the $\mathrm{C}-\mathrm{N}$ bond, and the bands located at 1045 and $780 \mathrm{~cm}^{-1}$ are due to the $\mathrm{N}-\mathrm{H}$ movement $[37,38]$. After the modification of HS particles by PPy polymer, the characteristic peaks of PPy are almost the same with some differences on the peak position and intensity. Further, the disappearance of the $-\mathrm{OH}$ bond after the addition of the polymer was seen, in which there is a strong chemical interaction between the residual - $\mathrm{OH}$ in the sand surface and the positively charged amine group of PPy. In addition, upon covering the HS particles by the polymer, the peak intensity attributed to Fe-O bond in the composite becomes very weak, as compared to the same bond in the pristine HS. The overall FTIR results confirm the occurrence of chemical interactions between the PPy active sites and the $\mathrm{Fe}_{2} \mathrm{O}_{3}$ present on the HS surface [39]. On the other hand, The FTIR spectrum of PANI, as presented in Figure 3b, shows many bands occurring at 1500 and $1555 \mathrm{~cm}^{-1}$, which are due to the $\mathrm{C}=\mathrm{C}$ stretching vibrations of the benzenoid and quinoid group, respectively. The bands at 1300 and $1153 \mathrm{~cm}^{-}$ ${ }^{1}$ are attributed to the stretching $\mathrm{C}-\mathrm{N}$ bond in the aromatic amine group, and the band located at $1123 \mathrm{~cm}^{-1}$ is due to bending of the $\mathrm{C}-\mathrm{H}$ bond located at the plane of the aromatic ring. Moreover, the band centred at $810 \mathrm{~cm}^{-1}$ is due to the deformation of the $\mathrm{C}-\mathrm{H}$ bond located in the out plane of the disubstituted benzene ring [31]. Likewise, the HS@PANI spectrum shows almost the same peaks characteristic of PANI. Further, the disappearance of the $-\mathrm{OH}$ bond after the addition of the polymer is clearly seen in the HS@PANI spectrum, in which there is a strong chemical interaction between the residual $-\mathrm{OH}$ in the surface of sand and the positively charged amine group of PANI. These results confirm the successful incorporation of hematite sand into the polymer matrix. The structures of the prepared catalysts were further investigated by Raman spectroscopy. The obtained Raman spectra of HS@PANI and HS@PPy are shown in Figures $\mathbf{3} \mathbf{c}$ and $\mathbf{d}$. As can be seen, the HS@PPy spectrum (Fig. 3c) indicates peaks 
occurring at 1575 and $1325 \mathrm{~cm}^{-1}$ which are attributed to the stretching of the backbone $\mathrm{C}=\mathrm{C}$, and the $\mathrm{C}-\mathrm{N}$, bonds, respectively. In addition, the peak located at $1055 \mathrm{~cm}^{-1}$ is due to the bending of the $\mathrm{C}-\mathrm{H}$ bond in the plane. Finally, the peak occurring at $971 \mathrm{~cm}^{-1}$ is due to the bending of the C-H bond [40]. Likewise, The Raman spectrum of HS@PANI (Fig. 3d) shows the characteristic main peaks of PANI due to the stretching $\mathrm{C}-\mathrm{N}$ bond centred in $1339 \mathrm{~cm}^{-1}$, the bending $\mathrm{C}-\mathrm{H}$ bond in the benzene ring centred $1259 \mathrm{~cm}^{-1}$, the bending $\mathrm{C}-\mathrm{H}$ bond in the quinoid ring centred at $1169 \mathrm{~cm}^{-1}$, and two characteristic peaks appear at $1500 \mathrm{~cm}^{-1}$ and $1600 \mathrm{~cm}^{-1}$ corresponding to the stretching of $\mathrm{C}-\mathrm{C}$ bond located in the benzene ring [41]. In addition, Raman bands characteristic of HS species, having low intensities, were also observed with at 610 and $655 \mathrm{~cm}^{-1}$, which may be due to the deposition of PANI and PPy layer on the surface of HS particles. The phase nature and the crystallinity of the HS@PANI and the HS@PPy composites were confirmed by XRD analyzes (Fig. 3e). The results indicate that the diffraction patterns of the HS particles, before and after their modification by PPy and PANI, remain the same, with small difference in the peaks Intensities. Therefore, most HS diffraction peaks can be perfectly indexed to the $\mathrm{Fe}_{2} \mathrm{O}_{3}$ structure, considering the nature of the sand used in our work. Hence, the XRD results show diffraction peaks occurring at $2 \theta=24.19^{\circ}, 33.20^{\circ}, 35.67^{\circ}$, $40.90^{\circ}, 49.49^{\circ}, 57.63^{\circ}$ corresponding to the iron oxide $\left(\mathrm{Fe}_{2} \mathrm{O}_{3}\right.$ ) (JCPDS No. 33-0664) $[34,39,42]$. The overall XRD data indicate that PPy and PANI polymers, covering the HS particles, are amorphous, contrary to the HS mineral which is highly crystallized. To assess the mass content in percent, of PANI and PPy in the HS@PANI and HS@PPy catalysts, the TGA curves were determined as shown in Figure 3f. Thus, as can be seen in this figure, the HS@PANI and HS@PPy composites have two major stages of mass losses. The first mass loss which appears in the temperature range $50^{\circ} \mathrm{C}-150^{\circ} \mathrm{C}$, corresponds to the loss of the adsorbed water, and it is expected to be $2 \%$ for both composites. The second mass loss is observed in the ranges 
$250-500{ }^{\circ} \mathrm{C}$, and it is indicative of the breakdown of PANI and PPy chains and the decomposition of the polymer matrix backbone. This second mass loss is about $15 \%$ for HS@PPy and 19 \% for HS@PANI [34,42,43]. After $505^{\circ} \mathrm{C}$, a plateau has been reached corresponding to the remaining HS material. Figure S3 shows the $\mathrm{N}_{2}$ adsorption-desorption isotherms of pure HS, and HS@PANI and HS@PPy composites, as measured at - $196{ }^{\circ} \mathrm{C}$, after degassing the samples at $80^{\circ} \mathrm{C}$ for 24 hours. The calculated BET surface areas were found to increase from $1.4087 \mathrm{~m}^{2} / \mathrm{g}$ (bare HS particles) to $4.7078 \mathrm{~m}^{2} / \mathrm{g}$ and $3.2648 \mathrm{~m}^{2} / \mathrm{g}$ after in-situ, PANI and PPy polymerization, respectively.

(a)

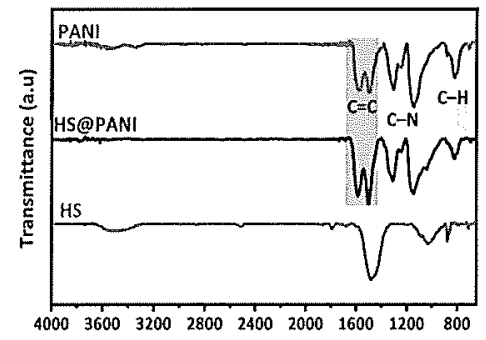

$\begin{array}{ll}400036003200 & 28002400 \\ \text { (b) } & \text { Wavenumber }\left(\mathrm{cm}^{-1}\right)\end{array}$

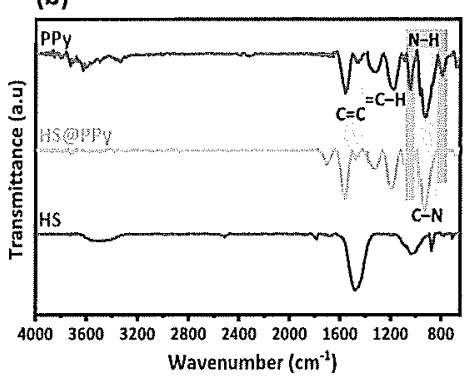

(c)

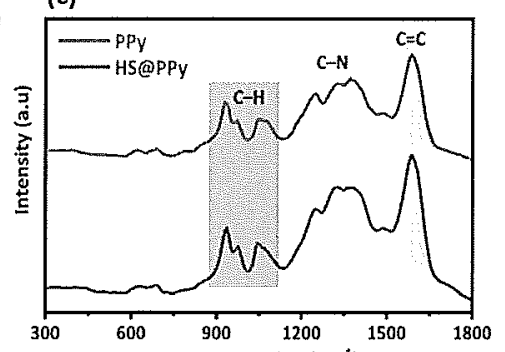

(d)

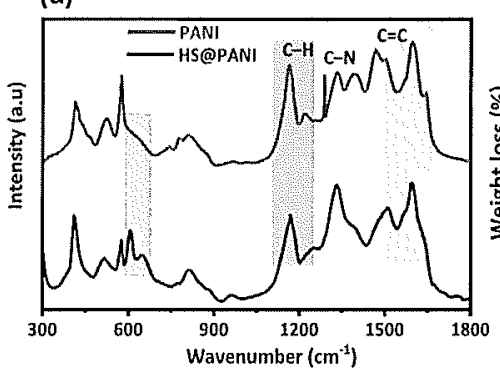

(e)
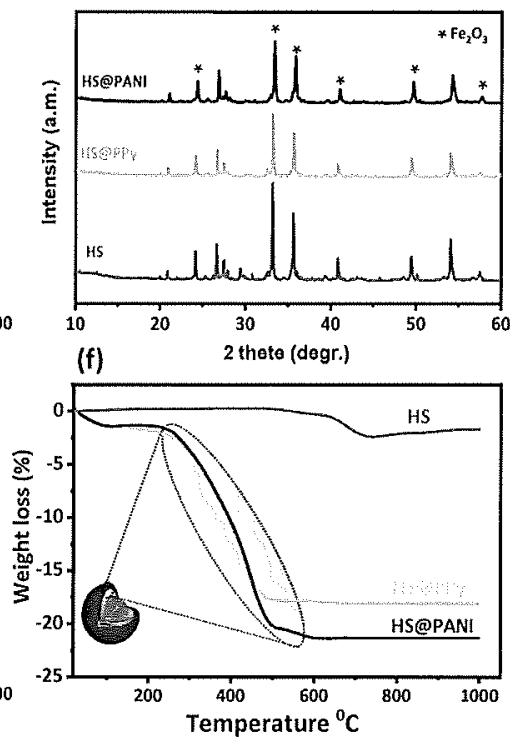

Fig. 3. FTIR spectra (a, b), Raman spectra (c, d), XRD spectra (e) and TG spectra(f) of HS, HS@PANI and HS@PPy

\subsection{Catalytic Performance.}

The catalytic oxidation performances of the prepared catalysts HS@PPy and HS@PANI, were evaluated for Orang $\mathrm{G}$ degradation by using PS as oxidant. The adsorption and catalytic 
performances are shown in Figure $\mathbf{4 a}$. The corresponding reaction rate constants are shown in Figure 4b. Firstly, the adsorption performances of HS@PANI and HS@PPy were tested. The results show that only about $15 \%$ and $20 \%$ OG were removed from water by adsorption on the catalyst, within 60 min, by using HS@PPy and HS@PANI, respectively. Secondly, blank experiments were carried out by using PS oxidant without catalyst to confirm its principal role, as well as, the resulting poor OG degradation rate. Such experiments have indicated that the PS alone was unable to oxidize OG in aqueous solution. These preliminary experiments allowed us to confirm the negligible contribution of the PS alone and the adsorption catalysts alone during the catalytic performance. After these control tests, the OG degradation efficiency, by using the HS/PS system, was found to be $30 \%$ indicating that HS particles alone might activate the PS to some extent. Surprisingly, when the HS particles were modified by PANI and PPy, increases in the catalytic oxidation efficiency of HS@PPy/PS and HS@PANI/PS systems were observed (reaching almost $100 \%$ in $60 \mathrm{~min}$ ). In addition, the corresponding reaction rate constant is significantly increased from $0.0054 \mathrm{~min}^{-1}$ for $\mathrm{HS}$ alone to 0.053 and 0.07 min $^{-1}$ for HS modified with PANI and PPy respectively. These better results are due to the synergistic effect and the combination of both HS and polymer materials. Initially, the formed polymer layer (PANI or PPy) on the HS surface allowed us a good fixation of the PS on the composite surface, as confirmed in the characterization part, and enhanced the production of the active species $\left(\mathrm{SO}_{4}{ }^{-*}, \mathrm{OH}^{\bullet}\right.$ and $\left.{ }^{1} \mathrm{O}_{2}\right)$. Afterwards, based on the oxidizing power of these reactive species, the $\mathrm{OG}$ molecules were then totally converted to $\mathrm{CO}_{2}$ and $\mathrm{H}_{2} \mathrm{O}$. More precisely, the use of conductive polymers, as it was characterized by structures rich in nitrogen atoms located in a conjugated system, provides a good performance PS activation. In addition, the $\mathrm{N}$ atoms act as active sites for the persulfate activation, as resulting from their higher electronegativity in comparison to carbon atoms $(\chi C=2.55<\chi N=3.04)$. This difference in the 
electronegativity between the nitrogen and the carbon atoms increases the electrons transfer capacity, and the charge density of the positively charged carbon adjacent, which behaves as an active site for adsorbing persulfate, cleaving hence the $\mathrm{O}-\mathrm{O}$ bond, and generating the ROS [12,44-47]. In addition, Rhodamine B (RhB) and Bisphenol A (BPA) were selected as other organic contaminants to confirm the performance of catalysts under the same experimental conditions. In Figure 4c the catalytic activity and the reaction rate constants are shown and indicate that both HS@PPy/PS and HS@PANI/PS systems can highly degrade the BPA and RhB after 120 min of contact time. These results confirm that HS@PANI and HS@PPy can be highly cleaning up wastewater from a wide range of toxic organic pollutants. In addition, to study the OG degradation under real conditions, the effect of tap water was performed for HS@PANI and HS@PPy systems. As shown in Figure 4d, within 60 min, the OG degradation amounts decreased from 100\% to 82\% and to 76\%, for HS@PANI and HS@PPy, respectively. These decreases were also confirmed by the degradation rate constants which decreased from 0.053 to $0.025 \mathrm{~min}^{-1}$ for HS@PANI, and from 0.070 to $0.027 \mathrm{~min}^{-1}$ for HS@PPy. The negative influence of the tap water medium on the OG removal rate could be due to the presence of chloride ions $\left(\mathrm{Cl}^{-}\right)$which deplete the radicals with a high level, and also to the presence of organic matter which decreases the OG degradation efficiency. The total organic carbon (TOC) analyzes were used to confirm the OG mineralization efficiency by using the HS@PPy/PS and HS@PANI/PS systems. As shown in Figure S4, the TOC removals are about 60\% and 57\%, by using HS@PANI and HS@PPy, respectively. These results confirm the catalytic degradation of $\mathrm{OG}$ into $\mathrm{CO}_{2}$ and $\mathrm{H}_{2} \mathrm{O}$, but also the presence of some intermediate molecules of $O G$ in the medium. To understand the origin of these excellent Fenton-like catalytic performances, the effects of experimental factors on the OG degradation were evaluated in Text S2 and Figure S5. 
(a)

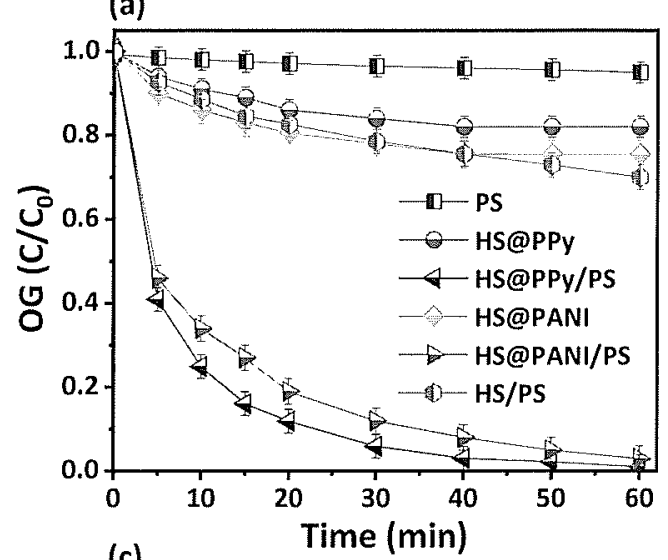

(c)

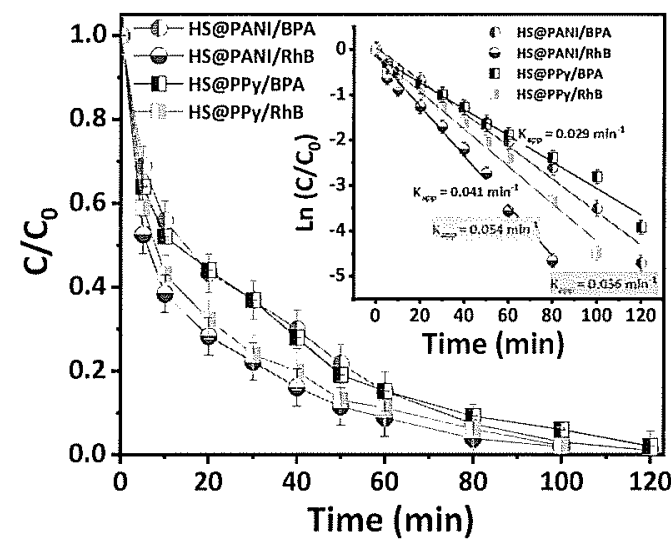

(b)

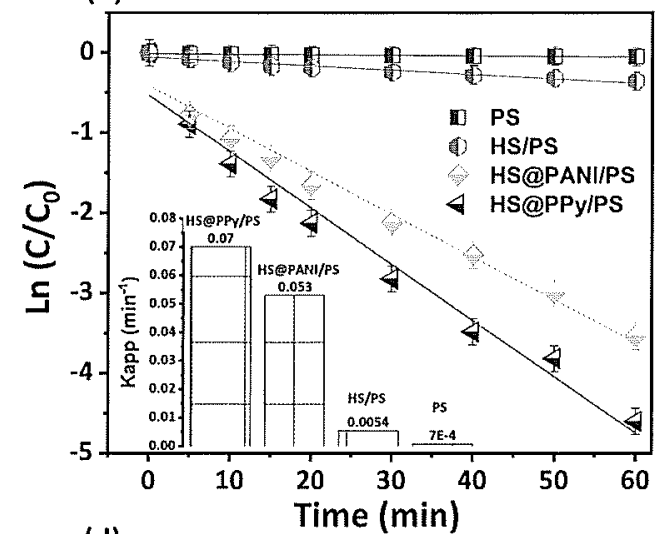

(d)

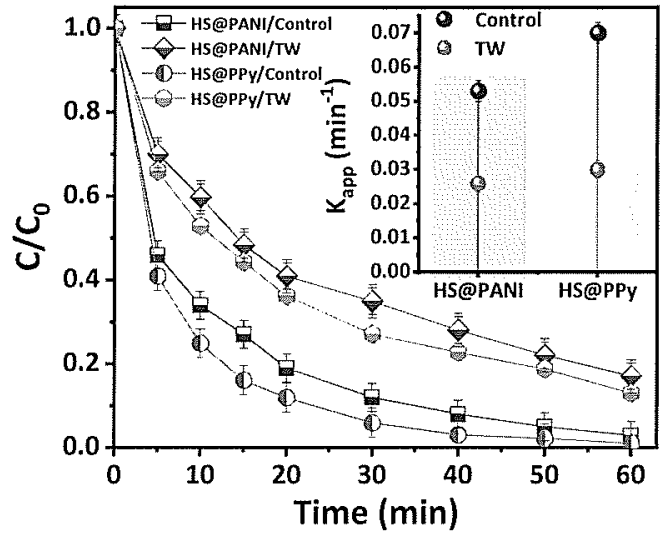

Fig. 4. Degradation of OG, and corresponding reaction rate constants, by using HS@PANI and HS@PPy (a, b) catalysts; Degradation of BPA and RhB: with HS@PANI and HS@PPy (c). Effect of tap water matrix, on the OG degradation (d). [Catalyst $]=0.2 \mathrm{~g} \mathrm{~L}^{-1},[\mathrm{OG}]=50 \mathrm{mg} \mathrm{L}^{-1},[\mathrm{BPA}]=$

$$
10 \mathrm{mg} \mathrm{L}^{-1},[\mathrm{RhB}]=20 \mathrm{mg} \mathrm{L}^{-1},[\mathrm{PS}]=4 \mathrm{mM}
$$

\subsection{ROS involved in the OG degradation.}

The radical quenching tests are performed to investigate the main reactive oxygen species (ROS) during the PS activation by using different catalysts. Ethanol (EtOH) and tertbutanol are used as selective radical scavengers to distinguish the contribution of $\mathrm{SO}^{-\bullet}$ and $\mathrm{OH} \bullet$ radicals in the PS system activation. Moreover, EtOH can deactivate both $\mathrm{OH}^{\bullet}(\mathrm{k}=9.7 \times$ $\left.10^{8} \mathrm{M}^{-1} \mathrm{~s}^{-1}\right)$ and $\mathrm{SO}_{4}{ }^{*-}\left(\mathrm{k}=2.5 \times 107 \mathrm{M}^{-1} \mathrm{~s}^{-1}\right)$, whereas TBA is used as a radical scavenger for $\mathrm{OH}^{\bullet}\left(k=3.8-7.6 \times 108 \mathrm{M}^{-1} \mathrm{~s}^{-1}\right)[1,6,7,17,48-50]$. As shown in Figure 5a, the catalytic efficiency 
of HS@PANI decreases slightly upon the addition of EtOH and TBA, from 100\% to $69 \%$ and to $88 \%$, respectively. The reaction rate constants decreased also from $0.053 \mathrm{~min}^{-1}$ (Control) to $0.017 \mathrm{~min}^{-1}(\mathrm{EtOH})$ and to $0.03 \mathrm{~min}^{-1}$ (TBA). However, the catalytic efficiency of HS@PPy was sharply reduced when EtOH and TBA were added. Thus, the reaction rate constants decreased from $0.07 \mathrm{~min}^{-1}$ (Control) to $0.017 \mathrm{~min}^{-1}$ (EtOH) and to $0.034 \mathrm{~min}^{-1}$ (TBA), respectively (Fig. 6a). These results suggest that $\mathrm{SO}_{4}{ }^{\cdot-}$ and $\mathrm{OH}^{*}$ play the primary role in the OG oxidation in the catalysts/PS systems. In addition to $\mathrm{OH}^{\bullet}$ and $\mathrm{SO}_{4}{ }^{*-}$, the nonradical specie $\left({ }^{1} \mathrm{O}_{2}\right)$ also plays a crucial role with a nonradical oxidation. The ${ }^{1} \mathrm{O}_{2}$ quenching experiments were conducted using $\mathrm{NaN}_{3}$ as a selective scavenger [6,7]. As shown in Figures $\mathbf{5} \mathbf{a}$ and $\mathbf{b}$, the activities of HS@PANI and HS@PPy decrease slightly with the addition of $\mathrm{NaN}_{3}$, and the reaction rate decreases from 0.07 to $0.016 \mathrm{~min}^{-1}$ for HS@PPy, and from 0.053 to $0.019 \mathrm{~min}^{-1}$ for HS@PANI. It is therefore concluded that ${ }^{1} \mathrm{O}_{2}$ is an important ROS during the PS activation in addition to $\mathrm{OH}^{\bullet}$ and $\mathrm{SO}_{4}{ }^{\bullet-}$. Moreover, PBN and TEMP trapped EPR tests further confirm the main reactive oxygen species (ROS). The PBN is an effective scavenger to inhibit $\mathrm{OH}^{\bullet}$ and $\mathrm{SO}_{4}{ }^{\circ}$ radicals, while TEMP could be used as a scavenger to probe the ${ }^{1} \mathrm{O}_{2}$ signal $[1,3,21,22,45,51-53]$. As shown in Figures $\mathbf{5} \mathrm{c}$ and d, in the absence of the catalysts, and when only the PS is added to the reaction solutions, no characteristic signals were detected upon the addition of the spin-trapping agents (PBN, TEMP). However, when HS@PANI and HS@PPy are added to the system, typical characteristic EPR spectra are detected, which represent the adduct signal PBN-SO ${ }_{4}{ }^{*-}$ and $\mathrm{PBN}-\mathrm{OH}{ }^{\bullet}[6,7]$. In addition, as shown in Figure $\mathbf{5 d}$, a characteristic three-line signal as a 1: 1: 1 triplet of TEMP agent was observed in the presence of the catalysts, which could be attributed to the TEMP${ }^{1} \mathrm{O}_{2}$ adduct. Therefore, these results demonstrate that the main reactive species in the current systems are $\mathrm{SO}_{4}{ }^{*-} \mathrm{OH}^{\bullet}$ from radical pathway and ${ }^{1} \mathrm{O}_{2}$ from nonradical pathway. 
(a)

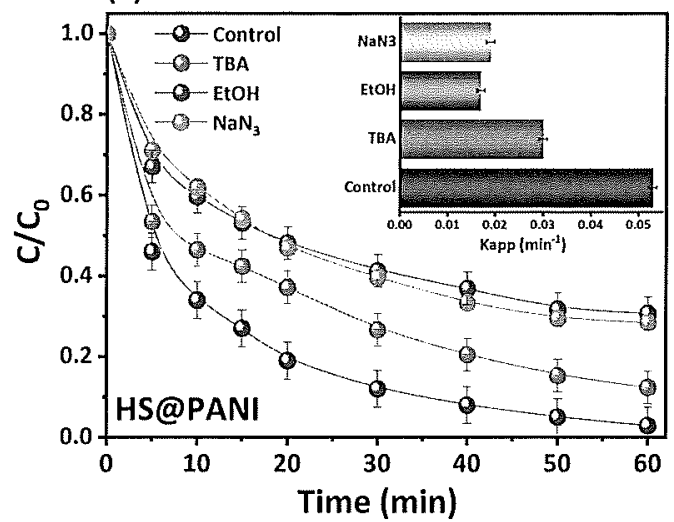

(c)

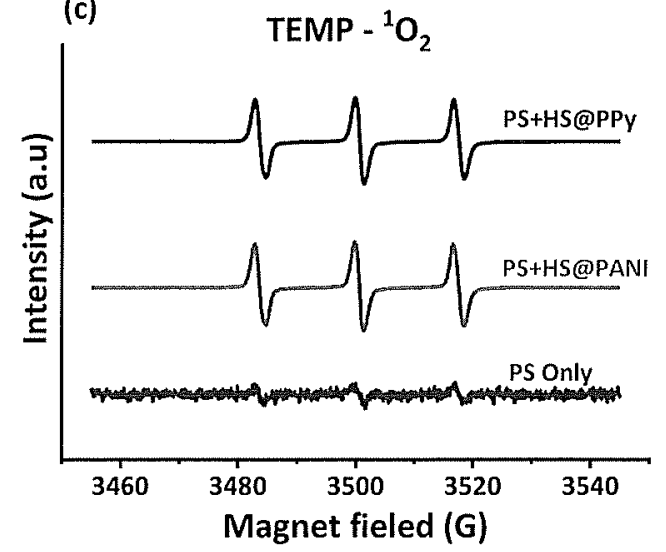

(b)

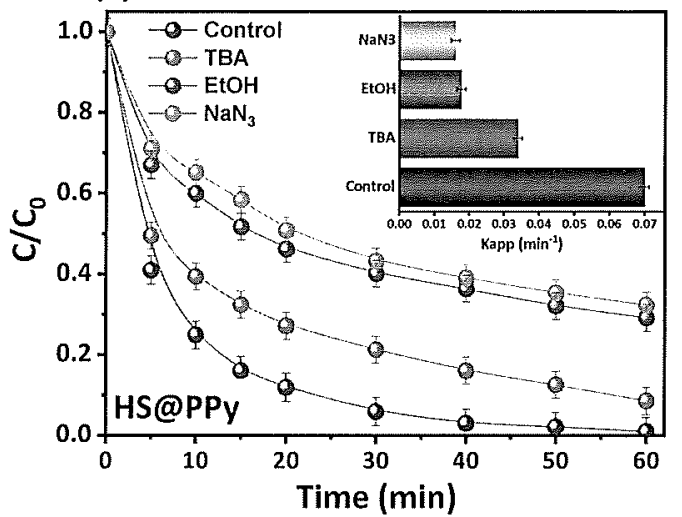

(d)

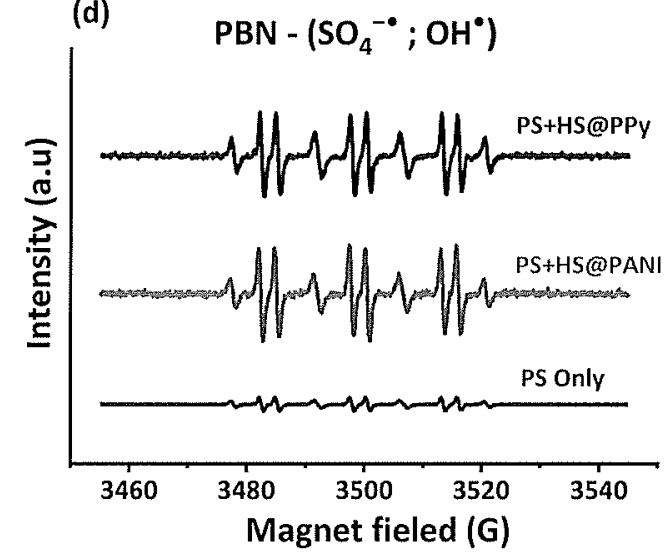

Fig. 5. Radical quenching results by using $\mathrm{EtOH}, \mathrm{TBA}$, and $\mathrm{NaN}_{3}$ as the scavengers in (a) HS@PANI/PS and (b) HS@PPy/PS. EPR spectra using (c) PBN and (d) TEMP, as the spintrapping agents for the catalyst/PS processes. (Experimental conditions: [Catalyst] $=0.2 \mathrm{~g} \mathrm{~L}^{-1}$, $[\mathrm{OG}]=50 \mathrm{mg} \mathrm{L}^{-1},[\mathrm{PS}]=4 \mathrm{mM},[\mathrm{EtOH}]=[\mathrm{TBA}]=1 \mathrm{M},\left[\mathrm{NaN}_{3}\right]=0.05 \mathrm{mM},[\mathrm{TEMP}]=[\mathrm{BPN}]=50$ $\mathrm{mM})$.

\subsection{Identification of surface-active sites and derived reaction mechanism.}

To confirm the contributions of the $\mathrm{HS}$ particles rich in $\mathrm{Fe}_{2} \mathrm{O}_{3}$ and the conductive polymers (PANI and PPy) rich in nitrogen, as well as, the synergistic effect during the PS activation, each material was studied separately.

- Role of HS core: Improving mediated electron transfer: The $\mathrm{Fe}_{2} \mathrm{O}_{3}$ metallic particles of HS plays a crucial role in enhancing the activating capacity of the PS (i.e. improving the 
overall electron transfer mediation capacity of the HS@Polymer). The results confirm that the incorporation of the $\mathrm{Fe}_{2} \mathrm{O}_{3}$ metallic particles of the $\mathrm{HS}$ into the polymer matrices significantly reduced the charge transfer resistance. The possible mechanism of the PS activation by the nucleus (HS) has been proposed for the heterogeneous OG degradation in the $\mathrm{HS} @$ Polymer/PS system. As shown in Schem. 1, the generation of $\mathrm{SO}_{4}{ }^{--}$radical by $\mathrm{Fe}_{2} \mathrm{O}_{3}$ was linked to the redox conversion of the $\equiv \mathrm{Fe}(I I I) / \equiv \mathrm{Fe}$ (II) ring as well as to the involvement of groups - $\mathrm{OH}$ on the surface (Eq. 1). Subsequently, $\mathrm{Fe}$ (II) or Fe(III) from $\mathrm{Fe}_{2} \mathrm{O}_{3}$ from the HS could activate $\mathrm{S}_{2} \mathrm{O}_{8}{ }^{2-}$ to produce $\mathrm{SO}_{4}{ }^{--}$and $\mathrm{SO}_{4}{ }^{2-}$ via the equations below. Newly formed $\mathrm{Fe}(\mathrm{II})-\mathrm{OH}$ coactivates PS with the inherent $\mathrm{Fe}(\mathrm{II})$ of $\mathrm{Fe}_{2} \mathrm{O}_{3}$ to produce $\mathrm{SO}_{4}{ }^{\circ-}$ and $\mathrm{Fe}(\mathrm{III})-\mathrm{OH}$ (Eqs 2 and 3) [5457]. These reactions result in the production of $\mathrm{OH}^{\bullet}$ radicals in two pathways (Eqs 4 and 5) [54-57]. As indicted, the role of HS in the HS@PANI and HS@PPy hybrid materials was observed to facilitate the electron transfer from the conjugated polymers to the PS. Therefore, the integration of metallic materials within the conductive nitrogen-rich polymers synergistically determines the chemical potential of the polymer surface, which effectively improve the PS activation.

$$
\begin{array}{ll}
\mathrm{Fe}(\mathrm{II})+2 \mathrm{H}_{2} \mathrm{O} \rightarrow \mathrm{Fe}(\mathrm{II})-\mathrm{OH}+\mathrm{H}_{3} \mathrm{O}^{+} & \mathrm{Eq}(1) \\
\mathrm{Fe}(\mathrm{II})-\mathrm{OH}+\mathrm{S}_{2} \mathrm{O}_{8}{ }^{2-} \rightarrow \mathrm{Fe}(\mathrm{III})-\mathrm{OH}+\mathrm{SO}_{4}{ }^{-}+\mathrm{SO}_{4}{ }^{2-} & \mathrm{Eq} \mathrm{(2)} \\
\mathrm{Fe}(\mathrm{III})-\mathrm{OH}+\mathrm{S}_{2} \mathrm{O}_{8}{ }^{2-} \rightarrow \mathrm{Fe}(\mathrm{II})-\mathrm{OH}+\mathrm{SO}_{4}{ }^{--}+\mathrm{SO}_{4}{ }^{2-} & \mathrm{Eq}(3) \\
\mathrm{SO}_{4}{ }^{--}+\mathrm{H}_{2} \mathrm{O} \rightarrow \mathrm{SO}_{4}{ }^{2-}+\mathrm{OH}^{+}+\mathrm{H}^{+} & \mathrm{Eq} \mathrm{(4)} \\
\mathrm{SO}_{4}{ }^{--}+\mathrm{OH}^{-} \rightarrow \mathrm{SO}_{4}{ }^{2-}+\mathrm{OH}^{-} & \mathrm{Eq}(5)
\end{array}
$$

- Effect of polymers coating: Efficiency improvement versus pathway switching: In the case of polymer materials containing nitrogen atoms, the stability of $\mathrm{N}$ atoms is a very important parameter to improve the stability and in the non-radical pathway in the 
catalysts/PS systems [31]. The loss of nitrogen in the catalysts/PS systems was considered to be a primary reason for the catalyst stability slow down and catalysts deactivation $[20,58,59]$. Therefore, the conducting polymers (PANI and PPy) used in this work are characterized by their stable $\mathrm{N}$ sites, and are suitable for long-lasting and efficiency catalytic reactions. Based on the experimental results, the possible mechanisms of the PS activation in the HS@PANI and HS@PPy, are directly linked by synergetic effect occurring between the PANI and the PPy shells and the HS as a metal core. The PS activation by HS@PANI and HS@PPy was expressed via radical and non-radical pathway as confirmed in the previous deactivation results (Scheme. 1). In fact, conducting polymers are the main sources of non-radical species during the catalytic reactions, as resulting from the electronegativity difference occurring between nitrogen and carbon atoms $(\chi C=2.55<\chi N=3.04)$. Further, the $N$ sites located in the conjugated system facilitate and increase the electrons transfer from the adjacent carbon atoms, creating hence positively charged carbons and leading to strongest active site for adsorption. Furthermore, the presence of the $\mathrm{N}$ atoms in the polymeric conjugated system creates a strong affinity towards the PS facilitating hence the O-O bond cleavage and generating the ROS for organic pollutants degradation. In more details, the hydrogen atoms transfer, occurring in the conjugated PANI and PPy systems, generates sufficient electrons that can highly cleave the O-O bond of $\mathrm{S}_{2} \mathrm{O}_{8}{ }^{2-}$ and release the $\mathrm{SO}_{4}{ }^{-}$radicals. Such reaction processes convert the conjugated $-\mathrm{NH}_{2}$ bond of PANI to $=\mathrm{NH}^{+}-($Eq. 6$)$, and the pyrrolic bond $(-\mathrm{NH}-)$ of PPy to $=\mathrm{N}^{\delta+} /=\mathrm{N}-($ Eq. 7).

$$
\begin{aligned}
& -\mathrm{NH}_{2}{ }^{+}-+\mathrm{S}_{2} \mathrm{O}_{8}{ }^{2-} \rightarrow=\mathrm{NH}^{+}-+\mathrm{SO}_{4}{ }^{-{ }^{-}}+\mathrm{SO}_{4}{ }^{2-} \\
& -\mathrm{NH}-+\mathrm{S}_{2} \mathrm{O}_{8}{ }^{2-} \rightarrow=\mathrm{N}^{\delta+} /=\mathrm{N}-+\mathrm{SO}_{4}{ }^{--}+\mathrm{SO}_{4}{ }^{2-}
\end{aligned}
$$$$
\mathrm{Eq}(6)
$$

On the other hand, the generated $\mathrm{SO}_{4}{ }^{*-}$ radicals can react with $\mathrm{H}_{2} \mathrm{O}$ molecules to yield the hydroxyl radical $\mathrm{OH}^{\bullet}$ (Eq. 8). 


$$
\mathrm{SO}_{4}{ }^{--}+\mathrm{H}_{2} \mathrm{O} \rightarrow \mathrm{SO}_{4}{ }^{2-}+\mathrm{OH}^{\bullet}+\mathrm{H}^{+}
$$

Moreover, the hydrogen and electrons transfer reactions occurring, at higher transient oxidation states, between the conjugated PANI and PPy systems and the $\mathrm{S}_{2} \mathrm{O}_{8}{ }^{2}$, lead to the break of the $\mathrm{O}-\mathrm{O}$ bonds of the PS, and the generation of the superoxide radicals $\mathrm{O}_{2}{ }^{\circ-}$ (Eqs. 9 and 10). It was reported that the $\mathrm{O}_{2}{ }^{-\bullet}$ radicals may be generated as intermediate product and that the ${ }^{1} \mathrm{O}_{2}$ may be produced from the oxidation of $\mathrm{O}_{2}{ }^{*-}$ (Eq. 11) [31].

$$
\begin{array}{ll}
=\mathrm{NH}^{+}-+\mathrm{S}_{2} \mathrm{O}_{8}{ }^{2-}+2 \mathrm{OH}^{-} \rightarrow-\mathrm{NH}_{2}{ }^{+}-+2 \mathrm{SO}_{4}{ }^{2-}+\mathrm{O}_{2}{ }^{--}+2 \mathrm{H}^{+} & \text {Eq (9) } \\
=\mathrm{N}^{\delta+} /=\mathrm{N}-+\mathrm{S}_{2} \mathrm{O}_{8}{ }^{2-}+2 \mathrm{OH}^{-} \rightarrow-\mathrm{NH}-+2 \mathrm{SO}_{4}{ }^{2-}+\mathrm{O}_{2}{ }^{--}+2 \mathrm{H}^{+} & \text {Eq (10) } \\
2 \mathrm{O}_{2}{ }^{--}+2 \mathrm{H}_{2} \mathrm{O} \rightarrow{ }^{1} \mathrm{O}_{2}+\mathrm{H}_{2} \mathrm{O}_{2}+2 \mathrm{OH}^{-} & \text {Eq (11) }
\end{array}
$$

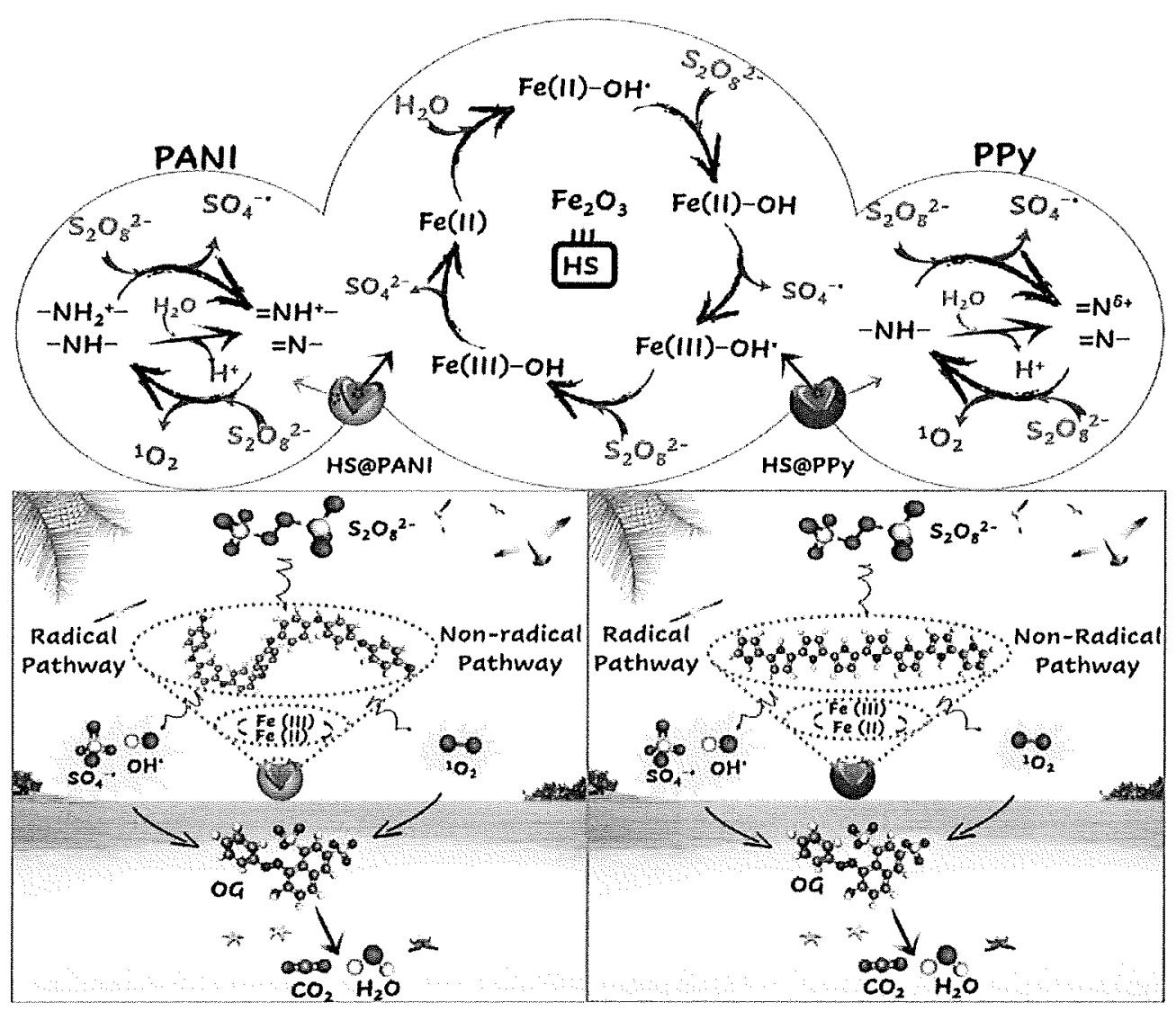

Scheme 1. Mechanism of the PS activation, on the HS@PANI and HS@PPy surfaces. 


\subsection{Catalyst chemical stability enhancement after the HS modification by the conducting polymers.}

The solution $\mathrm{pH}$ is one of the important factors that affect the stability of the prepared catalysts in the presence of PS, for the wastewater treatment $[53,57,60]$. Therefore, the metal coating on the HS surface by polymers prevents their leaching in the aqueous redox reactions, which has been known to be an essential factor responsible for the deactivation of metal catalysts. To confirm the protective role of these polymer (PANI and PPy) we studied the activation capacity of PS with HS@PPy and HS@PANI in a wide pH range (Fig. 6a). As shown in Figure, the OG was almost completely degraded within $60 \mathrm{~min}$ in the $\mathrm{pH}$ ranges $3-11$, indicating that both catalysts showed good performance for the activation of PS over a wide range of $\mathrm{pH}$ solution. However, when HS alone was used for the activation of PS, the higher removal rates were observed under acidic conditions, in contrast, when applying alkaline conditions (i.e. between $\mathrm{pH} 8$ and 11), a drastic reduction in removal efficiency was detected. These results suggest that the polymers act as protective layers, and/or they are related to the positive surface charge of both polymers as well as the protonation of nitrogen groups at the surface, which facilitated the adsorption of the negatively charged of $\mathrm{S}_{2} \mathrm{O}_{8}{ }^{2-}$. The overall data indicate that the HS@PANI and HS@PPy catalysts exhibit stable catalytic capacities over a wide $\mathrm{pH}$ range from 3 to 11 (Fig. $6 \mathrm{~b}$ ), which is by far among the widest $\mathrm{pH}$ range achieved by heterogeneous systems.

To clearly show the role of our molecular modification strategies by conductive polymers, we monitored the decrease in the PS activation efficiency of the pristine HS material, and the HS@PANI and the HS@PPy catalysts during the reuse of 5 cycles during catalytic degradation process (Fig. 6c). The SEM images and infrared spectra of HS@PANI and 
HS@PPy, after the recycling test remain almost the same with fresh HS@PANI and HS@PPy, which also prove that HS@PANI and HS@PPy are stable during the degradation process (Fig. S6). However, the pristine HS showed a greater decrease in performance, in comparison to the HS@PANI and the HS@PPy catalysts due to iron leaching after each cycle of use. Therefore, when using coated sand, the polymers play a protective role in decreasing the leaching of iron during the catalytic reaction. These results were well demonstrated in Figure 7c. As can be seen in this figure, during the last cycle, the efficiency of HS@PPy/PS and HS@PANI/PS decreased only 18 and 20\%, respectively. As the non-radical activation of persulfate involves insignificant consumption of the material, the constant decrease in the catalytic efficiency of HS@PANI and HS@PPy during the catalyst reuse could be attributed to the polymer surface and structural changes, including the deactivation of nitrogen atoms, iron leaching, adsorption of intermediates during the degradation of organic molecules and coverage of active sites on the surface of the catalyst. 
(a)

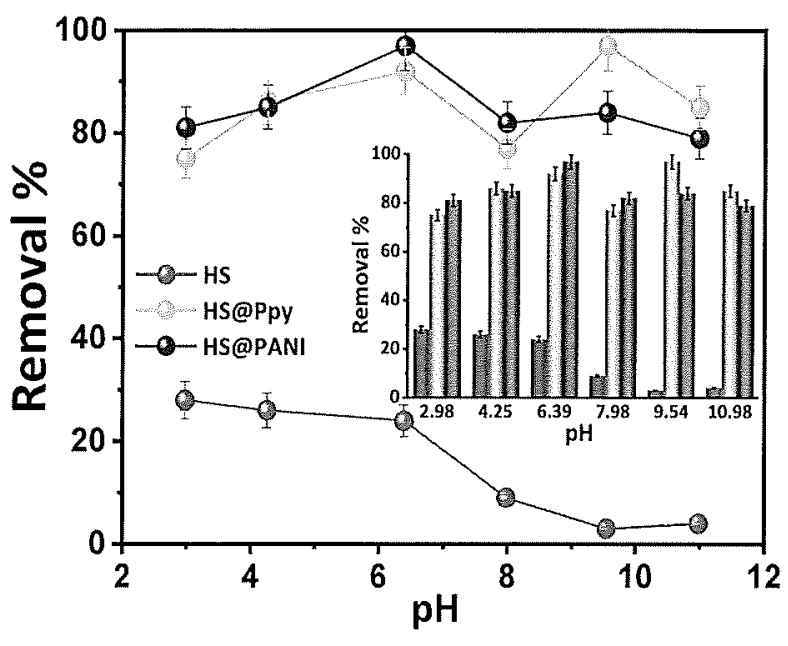

(b)

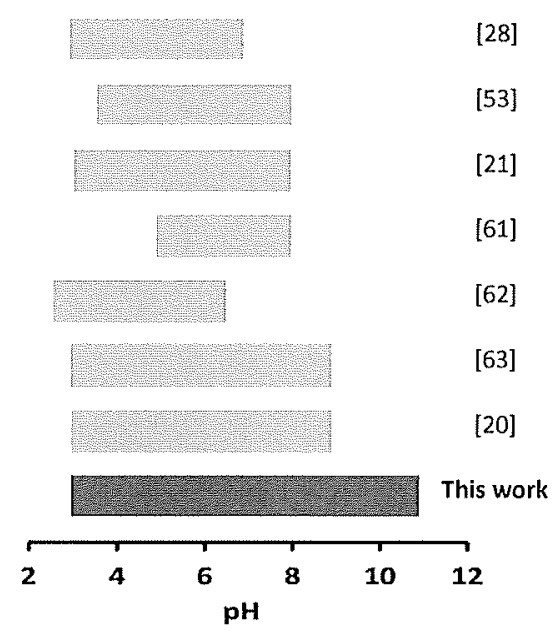

HS@PPy

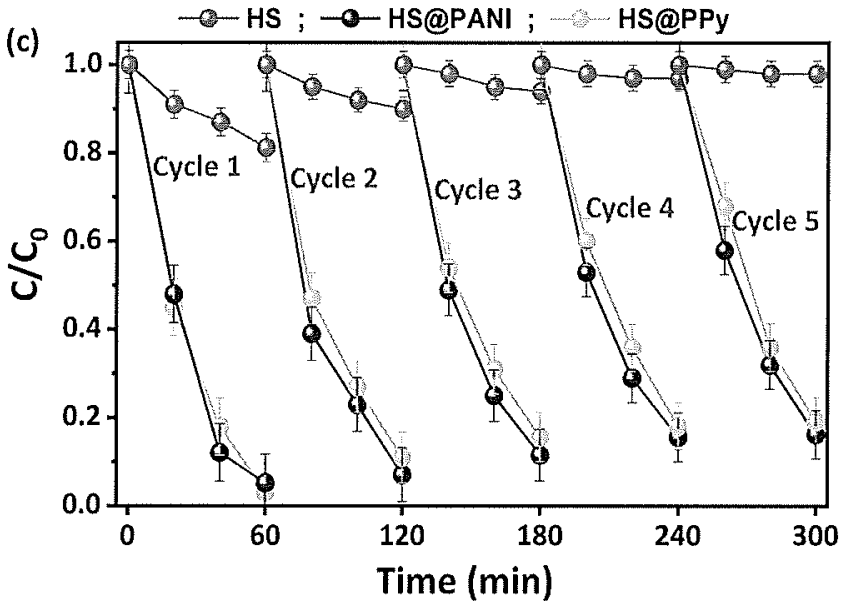

Fig. 7. Efficiency of HS, HS@PANI and HS@PPy for the OG degradation as monitored over broad pH ranges (a, b). Stability and reusability of HS, HS@PANI and HS@PPy (c). ([Catalyst]

$$
=0.2 \mathrm{~g} \mathrm{~L}^{-1},[\mathrm{OG}]=50 \mathrm{mg} \mathrm{L}^{-1},[\mathrm{PS}]=4 \mathrm{mM} \text { ) }
$$

\section{Conclusions}

In this study, we have investigated the effect of molecularly modified hematite sand/conductive polymer nanocomposites for ultrafast catalytic degradation of organic pollutants, by using persulfate as an activator. The encapsulation of HS particles into nitrogenrich polymer (PANI and PPy) matrices has led to synergistic catalytic effect occurring between 
the HS and the conducting polymers catalyst components, generating hence various reactive oxygen species. The data indicated that the nitrogen atoms, located in the conjugated chain of the conducting polymers covering the HS particles, played the main role in the adsorption and the activation of the persulfate, improving hence the organic pollutants degradation. Moreover, these polymeric systems generate both radical and non-radical species such as $\mathrm{SO}_{4}{ }^{*}, \mathrm{OH}^{*}$ and ${ }^{1} \mathrm{O}_{2}$. The use, of PANI and PPy in coating $\mathrm{HS}$ particles, was found to prevent iron leaching and polymer oxidation, avoiding hence secondary pollution, and offering an effective new strategy to develop green catalysts with nitrogen stable sites. Finally, the prepared catalysts were efficiently recovered after their uses, without significant loss. This work provides new insights into the synergetic effect occurring at the interface, between the HS core and the conducting polymer shell, for the PS activation and the ROS generation. In summary, the approach used in the present work provides an innovative method to produce high-quality and green catalysts in terms of environmental friendliness, cost effectiveness and comparable efficiency.

\section{CRediT author statement}

Abdellah Ait El Fakir: Writing - original draft, Conceptualization, Methodology, Data curation, Writing - review \& editing. Zakaria Anfar: Methodology, Data curation, Writing - review \& editing. Abdallah Amedlous: Methodology, Writing - review \& editing. Asma AMJLEF: Writing - review \& editing. Salaheddine Farsad: Writing - review \& editing. Amane Jada: Project administration, Supervision, Validation, Writing - review \& editing. Noureddine El Alem: Project administration, Supervision, Writing - review \& editing. 


\section{Acknowledgments}

This work was supported by Franco-Moroccan cooperation framework under both grant research projects APUR 2019 and CEDocs 2018 managed by Laboratory of Materials \& Environment (LME), Ibn Zohr University, Agadir - Morocco and the Institute of Materials Science of Mulhouse (IS2M), Haute Alsace University, Mulhouse - France. We thank, VAULOT Cyril (IS2M), VIDAL Loïc (IS2M), FIOUX Philippe (IS2M), MORLET - SAVARY Fabrice (IS2M) and GREE Simon (IS2M) for the analyses of samples by BET, TEM, SEM, XPS, EPR and FTIR-Raman, respectively.

\section{References}

[1] W. Ren, G. Nie, P. Zhou, H. Zhang, X. Duan, S. Wang, Environ. Sci. Technol. 54 (2020) 6438-6447.

[2] J. Yu, H. Feng, L. Tang, Y. Pang, G. Zeng, Y. Lu, H. Dong, J. Wang, Y. Liu, C. Feng, J. Wang, B. Peng, S. Ye, Prog. Mater. Sci. 111 (2020) 100654.

[3] P. Shao, S. Yu, X. Duan, L. Yang, H. Shi, L. Ding, J. Tian, L. Yang, X. Luo, S. Wang, Environ. Sci. Technol. 54 (2020) 8464-8472.

[4] B. Xing, J. Dong, G. Yang, N. Jiang, X. Liu, J. Yuan, Appl. Catal. A Gen. 602 (2020) 117714.

[5] X. Lin, Y. Ma, J. Wan, Y. Wang, Y. Li, Appl. Catal. A Gen. 589 (2020) 117307.

[6] Z. Anfar, A.A. El Fakir, M. Zbair, Z. Hafidi, A. Amedlous, M. Majdoub, S. Farsad, A. Amjlef, A. Jada, N. El Alem, Chem. Eng. J. 405 (2021) 126660. 
[7] Z. Anfar, A. Ait El Fakir, H. Ait Ahsaine, M. Zbair, S. Farsad, F. Morlet-Savary, A. Jada, N. El Alem, New J. Chem. 44 (2020) 9391-9401.

[8] L.W. Matzek, K.E. Carter, Chemosphere 151 (2016) 178-188.

[9] G. Fang, T. Zhang, H. Cui, D.D. Dionysiou, C. Liu, J. Gao, Y. Wang, D. Zhou, Environ. Sci. Technol. (2020) acs.est.0c06091.

[10] N. Jiang, H. Xu, L. Wang, J. Jiang, T. Zhang, Environ. Sci. Technol. 54 (2020) 1405714065.

[11] G.-X. Huang, C.-Y. Wang, C.-W. Yang, P.-C. Guo, H.-Q. Yu, Environ. Sci. Technol. 51 (2017) 12611-12618.

[12] X. Chen, X. Duan, W.-D. Oh, P.-H. Zhang, C.-T. Guan, Y.-A. Zhu, T.-T. Lim, Appl. Catal. B Environ. 253 (2019) 419-432.

[13] P. Sun, H. Liu, M. Feng, L. Guo, Z. Zhai, Y. Fang, X. Zhang, V.K. Sharma, Appl. Catal. B Environ. 251 (2019) 335-345.

[14] X. Duan, Z. Ao, H. Sun, S. Indrawirawan, Y. Wang, J. Kang, F. Liang, Z.H. Zhu, S. Wang, ACS Appl. Mater. Interfaces 7 (2015) 4169-4178.

[15] X. Duan, Z. Ao, H. Zhang, M. Saunders, H. Sun, Z. Shao, S. Wang, Appl. Catal. B Environ. $222(2018) 176-181$.

[16] P. Shao, J. Tian, F. Yang, X. Duan, S. Gao, W. Shi, X. Luo, F. Cui, S. Luo, S. Wang, Adv. Funct. Mater. 28 (2018) 1705295.

[17] X. Duan, H. Sun, Y. Wang, J. Kang, S. Wang, ACS Catal. 5 (2015) 553-559.

[18] P. Hu, H. Su, Z. Chen, C. Yu, Q. Li, B. Zhou, P.J.J. Alvarez, M. Long, Environ. Sci. Technol. 
$51(2017) 11288-11296$.

[19] X. Huo, P. Zhou, J. Zhang, Y. Liu, X. Cheng, Y. Liu, W. Li, Y. Zhang, J. Hazard. Mater. 391 (2020) 122055.

[20] H. Li, C. Shan, B. Pan, Environ. Sci. Technol. 52 (2018) 2197-2205.

[21] S. Wang, Y. Liu, J. Wang, Environ. Sci. Technol. 54 (2020) 10361-10369.

[22] E.-T. Yun, S.-W. Park, H.J. Shin, H. Lee, D.-W. Kim, J. Lee, Appl. Catal. B Environ. 279 (2020) 119360.

[23] W. Ma, N. Wang, Y. Du, T. Tong, L. Zhang, K.-Y. Andrew Lin, X. Han, Chem. Eng. J. 356 (2019) 1022-1031.

[24] L. Xu, B. Fu, Y. Sun, P. Jin, X. Bai, X. Jin, X. Shi, Y. Wang, S. Nie, Chem. Eng. J. 400 (2020) 125870.

[25] Y. Long, S. Li, Y. Su, S. Wang, S. Zhao, S. Wang, Z. Zhang, W. Huang, Y. Liu, Z. Zhang, Chem. Eng. J. 404 (2021) 126499.

[26] Y. Zhou, Y. Zhang, X. Hu, J. Colloid Interface Sci. 575 (2020) 206-219.

[27] N. Zhou, J. Zu, L. Yang, X. Shu, J. Guan, Y. Deng, D. Gong, C. Ding, M. Zhong, J. Colloid Interface Sci. 563 (2020) 197-206.

[28] P. Duan, Y. Qi, S. Feng, X. Peng, W. Wang, Y. Yue, Y. Shang, Y. Li, B. Gao, X. Xu, Appl. Catal. B Environ. 267 (2020) 118717.

[29] D. Ding, S. Yang, L. Chen, T. Cai, Chem. Eng. J. 392 (2020) 123725.

[30] J. Ye, J. Dai, C. Li, Y. Yan, Chem. Eng. J. (2020) 127805. 
[31] A. Ait El Fakir, Z. Anfar, A. Amedlous, M. Zbair, Z. Hafidi, M. El Achouri, A. Jada, N. El Alem, Appl. Catal. B Environ. 286 (2021).

[32] G. Sun, B. Dong, M. Cao, B. Wei, C. Hu, Chem. Mater. 23 (2011) 1587-1593.

[33] X.-F. Lu, X.-Y. Chen, W. Zhou, Y.-X. Tong, G.-R. Li, ACS Appl. Mater. Interfaces 7 (2015) $14843-14850$.

[34] Y. Zhang, D. Jiang, Y. Wang, T.C. Zhang, G. Xiang, Y.-X. Zhang, S. Yuan, Ind. Eng. Chem. Res. 59 (2020) 7554-7563.

[35] C. Wang, M. Yang, L. Liu, Y. Xu, X. Zhang, X. Cheng, S. Gao, Y. Gao, L. Huo, J. Colloid Interface Sci. 560 (2020) 312-320.

[36] K. Le, M. Gao, D. Xu, Z. Wang, G. Wang, W. Liu, F. Wang, J. Liu, Dalt. Trans. 49 (2020) 9701-9709.

[37] J. Tabačiarová, M. Mičušík, P. Fedorko, M. Omastová, Polym. Degrad. Stab. 120 (2015) 392-401.

[38] A. El Jaouhari, S. Ben Jadi, A. El Guerraf, M. Bouabdallaoui, Z. Aouzal, E.A. Bazzaoui, J.I. Martins, M. Bazzaoui, Synth. Met. 245 (2018) 237-244.

[39] F.A. Harraz, A.A. Ismail, S.A. Al-Sayari, A. Al-Hajry, J. Photochem. Photobiol. A Chem. 299 (2015) 18-24.

[40] A. El Jaouhari, A. El Asbahani, M. Bouabdallaoui, Z. Aouzal, D. Filotás, E.A. Bazzaoui, L. Nagy, G. Nagy, M. Bazzaoui, A. Albourine, D. Hartmann, Synth. Met. 226 (2017) 15-24.

[41] P.D. Bui, H.H. Tran, F. Kang, Y.-F. Wang, T.M. Cao, S.-J. You, N.H. Vu, V. Van Pham, ACS Appl. Nano Mater. 1 (2018) 5786-5794. 
[42] L. Wang, H. Yang, X. Liu, R. Zeng, M. Li, Y. Huang, X. Hu, Angew. Chemie Int. Ed. 56 (2017) 1105-1110.

[43] J. Liu, W. Zhou, L. Lai, H. Yang, S. Hua Lim, Y. Zhen, T. Yu, Z. Shen, J. Lin, Nano Energy 2 (2013) 726-732.

[44] S. Liu, C. Lai, B. Li, C. Zhang, M. Zhang, D. Huang, L. Qin, H. Yi, X. Liu, F. Huang, X. Zhou, L. Chen, Chem. Eng. J. 384 (2020) 123304.

[45] K. Zhu, Q. Bin, Y. Shen, J. Huang, D. He, W. Chen, Chem. Eng. J. 402 (2020) 126090.

[46] S. Ye, G. Zeng, X. Tan, H. Wu, J. Liang, B. Song, N. Tang, P. Zhang, Y. Yang, Q. Chen, X. Li, Appl. Catal. B Environ. 269 (2020) 118850.

[47] L. Tang, Y. Liu, J. Wang, G. Zeng, Y. Deng, H. Dong, H. Feng, J. Wang, B. Peng, Appl. Catal. B Environ. 231 (2018) 1-10.

[48] D. Ding, S. Yang, X. Qian, L. Chen, T. Cai, Appl. Catal. B Environ. 263 (2020) 118348.

[49] W. Ma, N. Wang, Y. Du, P. Xu, B. Sun, L. Zhang, K.-Y.A. Lin, ACS Sustain. Chem. Eng. 7 (2019) 2718-2727.

[50] H.-Y. Gao, C.-H. Huang, L. Mao, B. Shao, J. Shao, Z.-Y. Yan, M. Tang, B.-Z. Zhu, Environ. Sci. Technol. 54 (2020) 14046-14056.

[51] J. Yu, L. Tang, Y. Pang, G. Zeng, H. Feng, J. Zou, J. Wang, C. Feng, X. Zhu, X. Ouyang, J. Tan, Appl. Catal. B Environ. 260 (2020) 118160.

[52] J. Miao, W. Geng, P.J.J. Alvarez, M. Long, Environ. Sci. Technol. 54 (2020) 8473-8481.

[53] C. Liu, L. Liu, X. Tian, Y. Wang, R. Li, Y. Zhang, Z. Song, B. Xu, W. Chu, F. Qi, A. Ikhlaq, Appl. Catal. B Environ. 255 (2019) 117763. 
[54] F. Meng, M. Song, B. Song, Y. Wei, Q. Cao, Y. Cao, Chemosphere 243 (2020) 125322.

[55] H. Zheng, J. Bao, Y. Huang, L. Xiang, Faheem, B. Ren, J. Du, M.N. Nadagouda, D.D. Dionysiou, Appl. Catal. B Environ. 259 (2019) 118056.

[56] S. Guo, H. Wang, W. Yang, H. Fida, L. You, K. Zhou, Appl. Catal. B Environ. 262 (2020) 118250.

[57] L. Chen, Y. Huang, M. Zhou, K. Xing, W. Lv, W. Wang, H. Chen, Y. Yao, Chemosphere $250(2020) 126300$.

[58] Y. Gao, Y. Zhu, Z. Chen, C. Hu, ACS ES\&T Eng. (2020) acsestengg.0c00039.

[59] L. Peng, Y. Shang, B. Gao, X. Xu, Appl. Catal. B Environ. 282 (2021) 119484.

[60] H. Chi, J. Wan, Y. Ma, Y. Wang, M. Huang, X. Li, M. Pu, J. Hazard. Mater. 398 (2020) 123024.

[61] P. Duan, T. Ma, Y. Yue, Y. Li, X. Zhang, Y. Shang, B. Gao, Q. Zhang, Q. Yue, X. Xu, Environ. Sci. Nano 6 (2019) 1799-1811.

[62] S.-F. Jiang, L.-L. Ling, W.-J. Chen, W.-J. Liu, D.-C. Li, H. Jiang, Chem. Eng. J. 359 (2019) $572-583$.

[63] S. Liu, Z. Zhang, F. Huang, Y. Liu, L. Feng, J. Jiang, L. Zhang, F. Qi, C. Liu, Appl. Catal. B Environ. 286 (2021) 119921. 